Int. J. Mod. Phys. A (in press)

\title{
Theory of Neutral Particles: McLennan-Case Construct for Neutrino, its Generalization, and a New Wave Equation
}

\author{
D. V. Ahluwalia \\ Mail Stop H 846, Group P-25 \\ Los Alamos Meson Physics Facility \\ Los Alamos National Laboratory, Los Alamos, New Mexico 87545, USA \\ Internet address: AV@LAMPF.LANL.GOV
}

\begin{abstract}
Continuing our recent argument where we constructed a FNBWW-type spin-1 boson having opposite relative intrinsic parity to that of the associated antiparticle, we now study eigenstates of the Charge Conjugation operator. Based on the observation that if $\phi_{L}\left(p^{\mu}\right)$ transforms as a $(0, j)$ spinor under Lorentz boosts, then $\Theta_{[j]} \phi_{L}^{*}\left(p^{\mu}\right)$ transforms as a $(j, 0)$ spinor (with a similar relationship existing between $\phi_{R}\left(p^{\mu}\right)$ and $\Theta_{[j]} \phi_{R}^{*}\left(p^{\mu}\right)$; where $\Theta_{[j]} \mathbf{J} \Theta_{[j]}^{-1}=$ $-\mathbf{J}^{*}$ with $\Theta_{[j]}$ the well known Wigner matrix involved in the operation of time reversal) we introduce McLennan-Case type $(j, 0) \oplus(0, j)$ spinors. Relative phases between $\phi_{R}\left(p^{\mu}\right)$ and $\Theta_{[j]} \phi_{R}^{*}\left(p^{\mu}\right)$, and $\Theta_{[j]} \phi_{L}^{*}\left(p^{\mu}\right)$ and $\phi_{L}\left(p^{\mu}\right)$, turn out to have physical significance and are fixed by appropriate requirements. Explicit construction, and a series of physically relevant properties, for these spinors are obtained for spin- $1 / 2$ and spin- 1 culminating in the construction of a new wave equation and introduction of Dirac-like and Majorana-like quantum fields.
\end{abstract}

While in the case of gravitation the dynamical role played by space-time symmetries is manifest, the dynamical role played by space-time symmetries for other interactions is not always fully appreciated. This was recently emphasized in Ref [1]. Consider, as an example, quantum electrodynamics (QED). An essential element of the QED Lagrangian is the Dirac operator $\left(i \gamma^{\mu} \partial_{\mu}-m \mathbb{1}\right)$ for the charged fermion field $\Psi(x)$. The demand of covariance under the local gauge transformation, $\Psi(x) \rightarrow \exp (i \alpha(x)) \Psi(x)$, introduces a local $U(1)$ interaction via the vector potential $A_{\mu}(x)$. The basic building block, i.e., the operator $\left(i \gamma^{\mu} \partial_{\mu}-m \mathbb{1}\right)$, of the QED Lagrangian follows directly from the space-time symmetries and it contains significant information regarding the dynamical behavior and kinematical properties, such as relative intrinsic parities of the charged fermions and antifermions of spin- $1 / 2$, of the system. \& Without this, or similar, kinematical structure we would

1 To see how simply and deeply is the operator $\left(i \gamma^{\mu} \partial_{\mu}-m \mathbb{1}\right)$ connected with the space-time 
not even know how to formulate a principle of local gauge invariance for QED. Like mass and spin, [2 the concept of charge conjugation [3 and the associated notion of antiparticles arises from the Poincaré space-time symmetries.

The fact that QED, and presumably QCD, fermions are found in charge eigenstates is deeply connected with the structure of Dirac's $(1 / 2,0) \oplus(0,1 / 2)$ representation space. In fact, parity covariance is built into QED by building the kinematical structure of the theory on the Dirac's $(1 / 2,0) \oplus(0,1 / 2)$ field rather than the Weyl's $(1 / 2,0)$ or $(0,1 / 2)$ field. From Majorana's work [6] one knows that Dirac's construct is not the only construct possible for the $(1 / 2,0) \oplus(0,1 / 2)$ quantum field; moreover, we shall see that there are additional possibilities. An important reformulation of Majorana's work was undertaken by McLennan and Case [9, 10, in 1957. Here we shall extend the McLennan-Case formulation and generalize it to spin-1 (and higher). While considering spin-1, a surprising conclusion is reached: Within the framework of the formalism developed in this paper, there are no self-charge conjugate spinors in the $(1,0) \oplus(0,1)$ representation space. Fundamentally

symmetries, the reader may first refer to Sec. 2.3 of Ryder's book [2], next study Ref. [3], and finally refer to the text bracketed between Eqs. (1) and (17) of Ref. [4], which provides some of the missing details of the previous two references. Here is the argument in brief: Refer to Eqs. (1a) and (1b), and the surrounding definitions, of this paper and set $\boldsymbol{J}=\boldsymbol{\sigma} / 2$. Next, note that spinors [implied by the arguments based on parity symmetry and that Lorentz group is essentially $\left.S U_{R}(2) \otimes S U_{L}(2)\right]$

$$
\psi\left(p^{\mu}\right) \equiv\left(\begin{array}{c}
\phi_{R}\left(p^{\mu}\right) \\
\phi_{L}\left(p^{\mu}\right)
\end{array}\right)
$$

turn out to be of crucial significance in constructing a field $\Psi(x)$ that describes eigenstates of the Charge operator, $Q$, if $\phi_{R}\left(\stackrel{\circ}{p}^{\mu}\right)= \pm \phi_{L}\left(\stackrel{\circ}{p}^{\mu}\right)$ (otherwise physical eigenstates are no longer charge eigenstates). We call $\phi_{R}\left(\stackrel{\circ}{p}^{\mu}\right)= \pm \phi_{L}\left(\stackrel{\circ}{p}^{\mu}\right)$, the "Ryder-Burgard relation" (see Ref. [2] and footnote [11]). Next couple the Ryder-Burgard relation with Eqs. (1a) and (1b) to obtain (useful identities: $\cosh (\boldsymbol{\sigma} \cdot \boldsymbol{\varphi})=\mathbb{1} \cosh \varphi, \sinh (\boldsymbol{\sigma} \cdot \boldsymbol{\varphi})=\boldsymbol{\sigma} \cdot \widehat{\boldsymbol{p}} \sinh \varphi, \cosh \varphi=E / m, \sinh \varphi=|\boldsymbol{p}| / m)$

$$
\left(\begin{array}{cc}
\mp m \mathbb{1} & p_{0}+\boldsymbol{\sigma} \cdot \boldsymbol{p} \\
p_{0}-\boldsymbol{\sigma} \cdot \boldsymbol{p} & \mp m \mathbb{1}
\end{array}\right) \psi\left(p^{\mu}\right)=0 .
$$

Introducing $\psi(x) \equiv \psi\left(p^{\mu}\right) \exp (\mp i p \cdot x)$ and letting $p_{\mu} \rightarrow i \partial_{\mu}$, the above equation becomes: $\left(i \gamma^{\mu} \partial_{\mu}-m \mathbb{1}\right) \psi(x)=0$. This is the Dirac equation for spin- $1 / 2$ particles with $\gamma^{\mu}$ in the Weyl/Chiral representation. Similarly, one can obtain wave equations and thus a complete kinematic structure and the associated dynamical consequences for other Dirac-like $(j, 0) \oplus(0, j)$ spinors $\psi\left(p^{\mu}\right)$ and quantum fields $\Psi(x)$. Also see footnote 7 .

${ }^{2}$ Recall that mass and spin are intimately related with the two Casimir invariants of the Poincaré group. The two Casimir invariants are $C_{1} \equiv P^{\mu} P_{\mu}$, and $C_{2} \equiv W^{\mu} W_{\mu}$ with the Pauli-Lubanski pseudovector defined as $W_{\mu} \equiv-(1 / 2) \epsilon_{\mu \nu \rho \sigma} J^{\nu \rho} P^{\sigma}[5]$.

${ }^{3}$ The positive- and negative-energy solutions associated with the Dirac equation purely at the kinematical level essentially suffice to suggest the concept of Charge Conjugation. 
new wave equations, non-unitarily connected to Dirac (for spin-1/2) or modified Weinberg equation (for spin-1), will be presented.

Some of the constructs presented may appear familiar and well known. For example, half of the type II $(1 / 2,0) \oplus(0,1 / 2)$ spinors presented below are identical with Majorana spinors (Ref. [11], p. 20) The remaining half of the type II spinors for spin-1/2, introduced as anti-self charge conjugate counterparts of Majorana spinors, are needed to obtain a complete set ("complete" in the mathematical sense). The extension to higher spins is accomplished by recognizing that the "magic of the Pauli matrices", Ref. [11] Eq. 1.4.24, is in fact a special example of the relation $\Theta_{[j]} \mathbf{J} \Theta_{[j]}^{-1}=-\mathbf{J}^{*}$ with $\Theta_{[j]}$ the well known Wigner matrix involved in the operation of time reversal. To my knowledge, no equation of motion for these spinors in their $2(2 j+1)$-element form exists in literature. This fact in my view has prevented the full exploitation, and understanding of the precise physical content, of a field theory based on Majorana (and the associated anti-self charge conjugate spinors) spinors. It is hoped that the fundamentally new wave equation derived in this work will remedy the existing situation and lead to a deeper understanding of the work initiated by McLennan and Case. f Also the facts that the type II spinors cannot be helicity eigenspinors, or that their properties are unusual under the operation of parity, or that introducing gauge interactions via the standard minimal substitution for such particles is impossible, are, to the best of my knowledge, all new results.

It is hoped that the formalism developed here will provide, if so necessitated by future theoretical and (or) experimental reasons, a from-the-first-principle point of departure for the kinematical and dynamical understanding of neutrinos and photinos. For instance, if the phenomenon of neutrino oscillations is established [13] then one will be forced to express the weak eigenstates as a linear superposition of mass eigenstates. In this context the question would arise if the mass eigenstates can have a description beyond the well known Dirac and Majorana states and on what theoretical/experimental grounds one will choose the various mass eigenstates in the superposition. Experimental results, in general, cannot be insensitive to such choices and the P, CP, transformation of the states will depend on the choices made in the theoretical models.

To establish the setting of the ideas it is perhaps appropriate to orient the discussion by making a few brief observations on the subject. It was in 1927 that Wigner introduced the notion of parity in quantum mechanics [14]. In his celebrated paper of 1939 [5] and his 1962 collaborative work with Bargmann and Wightman, without reference to any wave equations, Wigner [15] classified quantum field theories on the basis of their behavior under continuous space-time symmetries and the operations of discrete symmetries of Parity, Time Reversal and Charge Conjugation. Since the transformation properties of quantum mechanical states do not directly invoke a wave equation and since the operation of Charge Conjugation cannot be determined without exploiting an appropriate wave equation, a specific connection between the Lorentz group representations and their behavior under the operations of Parity, Time Reversal and Charge Conjugation had remained essentially unexplored beyond spin-1/2. While Weinberg [16] did undertake a general study of this connection in 1964 for the $(j, 0) \oplus(0, j)$ representation space, various details escaped notice until a recent

4 The Jehle [12] type equations that appear in Refs. 9, 10] for spin-1/2 (and its simple generalization to higher spins) while consistent with our work, are far from being wave equations for the $2(2 j+1)$-element $(j, 0) \oplus(0, j)$ spinors of type-II introduced in this work. The exact meaning of this remark will become clear as we proceed. 
publication [3]. In Ref. [3] it was shown that the $(1,0) \oplus(0,1)$ representation space is a concrete realization of a FNBWW (Foldy-Nigam-Bargmann-Wightman-Wigner Đ) type quantum field theory in which a boson and its antiboson carry opposite relative intrinsic parities. In Ref. [3] we confined our attention to particles that are eigenstates of the Charge operator. In this paper, by going beyond the eigenstates of the Charge operator, I provide a further instance of establishing the kinematical structure of a field theory upon the relevant space-time symmetries.

To avoid possible confusion, we parenthtically note that the representation space under consideration in Weinberg's work [16] and the present work is the $(j, 0) \oplus(0, j)$ representation space. Contrary to the canonical wisdom, we have recently established [3] that certain fundamental aspects of a quantum field theory are deeply connected with the representation space one chooses to describe a spin- $j$ particle. For description of particles outside the $(j, 0) \oplus(0, j)$ representation space there are well known works and some of the fundamental papers are catlogued in Ref. [17]

\section{Two Types of $(j, 0) \oplus(0, j)$ Spinors}

To define our conventions and notation, following the 1939 classic work of Wigner [5 we note that, without reference to any wave equation, the $(j, 0)$ and $(0, j)$ spinors, in the notation of our earlier work [3], under Lorentz transformations transform as

$$
\begin{array}{ll}
(j, 0): & \phi_{R}\left(p^{\mu}\right)=\Lambda_{R}\left(p^{\mu} \leftarrow \stackrel{p}{p}^{\mu}\right) \phi_{R}\left(\stackrel{\circ}{p}^{\mu}\right), \\
(0, j): & \phi_{L}\left(p^{\mu}\right)=\Lambda_{L}\left(p^{\mu} \leftarrow \stackrel{p}{p}^{\mu}\right) \phi_{L}\left(\stackrel{p}{p}^{\mu}\right) .
\end{array}
$$

In the above expressions $\stackrel{\circ}{\mu}^{\mu}$ refers to the energy-momentum four vector associated with the particle, of mass $m$, at rest; and the boost parameter $\varphi$ is defined as in Eq. (3) of Ref. [3]. The Wigner boosts for the $(j, 0)$ and $(0, j)$ spinors are

$$
\Lambda_{R}\left(p^{\mu} \leftarrow \stackrel{\circ}{p}^{\mu}\right)=\exp \left(i \mathbf{K}^{(\mathbf{j}, \mathbf{0})} \cdot \boldsymbol{\varphi}\right), \quad \Lambda_{L}\left(p^{\mu} \leftarrow \stackrel{\circ}{p}^{\mu}\right)=\exp \left(i \mathbf{K}^{(\mathbf{0}, \mathbf{j})} \cdot \boldsymbol{\varphi}\right)
$$

For the $(j, 0)$ representation space $\mathbf{B} \equiv 2^{-1}(\mathbf{J}-i \mathbf{K})$ equals zero, and therefore $\mathbf{K}^{(\mathbf{j}, \mathbf{0})}=-i \mathbf{J}$. Similarly, for the $(0, j)$ representation space $\mathbf{A} \equiv 2^{-1}(\mathbf{J}+i \mathbf{K})$ equals zero, and therefore $\mathbf{K}^{(\mathbf{0}, \mathbf{j})}=$ $+i$ J. Consequently,

$$
\Lambda_{R}\left(p^{\mu} \leftarrow \stackrel{\circ}{p}^{\mu}\right)=\exp (\mathbf{J} \cdot \boldsymbol{\varphi}), \quad \Lambda_{L}\left(p^{\mu} \leftarrow \stackrel{\circ}{p}^{\mu}\right)=\exp (-\mathbf{J} \cdot \boldsymbol{\varphi}) .
$$

Here, $\mathbf{J}$ are the standard spin- $j$ matrices with $J_{z}$ diagonal. Having established our conventions and notation, we now note that $\left[\Lambda_{L, R}\left(p^{\mu} \leftarrow \stackrel{\circ}{\mu}^{\mu}\right)\right]^{-1}=\left[\Lambda_{R, L}\left(p^{\mu} \leftarrow \grave{p}^{\mu}\right)\right]^{\dagger}$ and the fact that Wigner's operator $\Theta_{[j]}$ for spin- $j$ has the property $\Theta_{[j]} \mathbf{J} \Theta_{[j]}^{-1}=-\mathbf{J}^{*}$ implies that if $\phi_{L}\left(p^{\mu}\right)$ transforms as

\footnotetext{
${ }^{5}$ After the publication of Ref. [3], and after much of the present work was already in draft form, we learned that ideas similar to those of Bargmann, Wightman, and Wigner were put forward previously and independently by Foldy and Nigam [18,19]. I thank Dr. Zurab K. Silagadze for bringing Foldy's paper to my attention. Professor Foldy brought Ref. [19] to my attention where, in essence, Nigam and Foldy constructed a quantum field theory where a spin- $1 / 2$ fermion and its antifermion have same relative intrinsic parity. It is my pleasure to thank Professors Foldy and Nigam for conversations and correspondence.
} 
a $(0, j)$ spinor under Lorentz boosts, then $\Theta_{[j]} \phi_{L}^{*}\left(p^{\mu}\right)$ transforms as a $(j, 0)$ spinor. Similarly, if $\phi_{R}\left(p^{\mu}\right)$ transforms as a $(j, 0)$ spinor under Lorentz boosts, then $\Theta_{[j]} \phi_{R}^{*}\left(p^{\mu}\right)$ transforms as a $(0, j)$ spinor. Wigner's operator is defined as $\left(\Theta_{[j]}\right)_{\sigma, \sigma^{\prime}}=(-1)^{j+\sigma} \delta_{\sigma^{\prime},-\sigma}$ with $\sigma$ and $\sigma^{\prime}$ as eigenvalues of J. By definition $\Theta_{[j]}$ is real. For spin- $1 / 2$ and spin-1 it reads:

$$
\Theta_{[1 / 2]}=\left(\begin{array}{cc}
0 & -1 \\
1 & 0
\end{array}\right), \quad \Theta_{[1]}=\left(\begin{array}{ccc}
0 & 0 & 1 \\
0 & -1 & 0 \\
1 & 0 & 0
\end{array}\right)
$$

These observations allow us to introduce two types of $(j, 0) \oplus(0, j)$ spinors. The first type,

$$
\psi\left(p^{\mu}\right) \equiv\left(\begin{array}{c}
\phi_{R}\left(p^{\mu}\right) \\
\phi_{L}\left(p^{\mu}\right)
\end{array}\right)
$$

to be referred as spinors of Type I. The second type,

$$
\lambda\left(p^{\mu}\right) \equiv\left(\begin{array}{c}
\left(\zeta_{\lambda} \Theta_{[j]}\right) \phi_{L}^{*}\left(p^{\mu}\right) \\
\phi_{L}\left(p^{\mu}\right)
\end{array}\right), \quad \rho\left(p^{\mu}\right) \equiv\left(\begin{array}{c}
\phi_{R}\left(p^{\mu}\right) \\
\left(\zeta_{\rho} \Theta_{[j]}\right)^{*} \phi_{R}^{*}\left(p^{\mu}\right)
\end{array}\right),
$$

to be referred as spinors of Type II. In Eq. (6) $\zeta_{\lambda}$ and $\zeta_{\rho}$ are phase factors that are yet to be fixed.

We now begin with a general study of some of the properties of these spinors and quantum field theories based on these spinors. The arguments that follow can be made for any spin, but since spin-1/2 and spin-1 are of special prominence in phenomenological descriptions, we confine ourselves to these two spins. For spin-1/2 the operation of charge conjugation and parity ("space inversion" f ) on the $(1 / 2,0) \oplus(0,1 / 2)$-representation-space spinors is given by [11,20]

$$
S_{[1 / 2]}^{c}=e^{i \vartheta_{[1 / 2]}^{c}}\left(\begin{array}{cc}
0 & i \Theta_{[1 / 2]} \\
-i \Theta_{[1 / 2]} & 0
\end{array}\right) \mathcal{K} \equiv \mathcal{C}_{[1 / 2]} \mathcal{K}, \quad S_{[1 / 2]}^{s}=e^{i \vartheta_{[1 / 2]}^{s}}\left(\begin{array}{cc}
0 & \mathbb{1}_{2} \\
\mathbb{1}_{2} & 0
\end{array}\right)=e^{i \vartheta_{[1 / 2]}^{s}} \gamma^{0},
$$

where $\mathcal{K}$ is the operation of complex conjugation, and $\mathbb{1}_{2}$ is a $2 \times 2$ identity matrix. While this result is well known, the correct counterpart of results $(7)$ for the $(1,0) \oplus(0,1)$ representation space required a careful study. A detailed analysis was recently presented in Ref. [3]; here we simply need the result. These results read:

$$
S_{[1]}^{c}=e^{i \vartheta_{[1]}^{c}}\left(\begin{array}{cc}
0 & \Theta_{[1]} \\
-\Theta_{[1]} & 0
\end{array}\right) \mathcal{K} \equiv \mathcal{C}_{[1]} \mathcal{K}, \quad S_{[1]}^{s}=e^{i \vartheta_{[1]}^{s}}\left(\begin{array}{cc}
0 & \mathbb{1}_{3} \\
\mathbb{1}_{3} & 0
\end{array}\right)=e^{i \vartheta_{[1]}^{s}} \gamma_{00}
$$

Note that neither $S_{[1 / 2]}^{c}$ nor $S_{[1]}^{c}$ are unitary (or even linear). This fact, besides others, necessitates building a quantum field theory (the so-called "second quantized theory") containing a field operator, $\Psi_{[j]}(x)$ expanded in terms of the above spinors. The operation of charge conjugation is then defined via a unitary operator $U_{[j]}^{c}$ such that 19

$$
U_{[j]}^{c} \Psi_{[j]}(x)\left(U_{[j]}^{c}\right)^{-1}=\mathcal{C}_{[j]} \Psi_{[j]}^{\dagger}(x)
$$

\footnotetext{
${ }^{6}$ As usual, the operation of parity in space-time is defined as $\mathcal{P}: \quad \mathbf{x} \rightarrow-\mathbf{x}$ and $t \rightarrow t$.
} 
For Majorana fields, internal consistency of the theory requires $\vartheta^{s}$ and $\vartheta^{c}$ to be constrained. However, incorporating such constrained phase factors can be done as needed and we shall choose $\vartheta^{c}=0$ so as to stay as close as possible to Ramond's 11 discussion on the subject. A further note of caution is in order. Using the type-I spinors, one may construct a Majorana field operator for any spin [21]. In this formalism the field describes states of a particle that are simultaneously eigenstates of the Parity operator (with imaginary eigenvalues [7]) and the Charge Conjugation operator. But how can that be? For spin-1/2, for instance, the usual text-book expressions for $U^{s}$ and $U^{c}$ do not commute! This apparent inconsistency is resolved in the careful analysis of the $U^{s}$ and $U^{c}$ operators by Nigam and Foldy [19] which reveals that $U^{s}$ is in fact a function of the Charge operator. For self-charge conjugate fields, if one makes the additional assumption that the Charge operator acting on a self-charge-conjugate state yields zero (an assumption certainly not valid for charged particles, because then simultaneous eigenstates of the Charge Conjugation operator and Charge operator are not possible), one finds that the commutator of the $U^{s}$ and $U^{c}$ vanishes upon acting on Majorana states. The commutativity/anticommutativity of $U^{s}$ and $U^{c}$ operators is a function of the Charge operator with formal and phenomenological consequences.

When one constructs a quantum field based on the $(1,0) \oplus(0,1)$ spinors of type I, one finds that the Fock space operators for charge eigenstates that determine transformation of physical states under the operation of Charge Conjugation, $U_{[1]}^{c}$, and Parity, $U_{[1]}^{s}$, anticommute. This results in a quantum field theory of spin-1 bosons where a boson and its anti-boson carry opposite relative intrinsic parity. For this aspect of the kinematic structure we refer the reader to our recent publication [3]. In the rest of the paper we confine our attention to spinors of type II, and the quantum field theory that is built upon them.

We begin with the question: What physical requirement can fix the phase factors $\zeta$ that appear in the definition of the spinors of Type II ? We do not, at the moment, investigate all possible physically relevant requirements but confine to the most obvious ones.

For spin-1/2, we find that the requirement of self/anti-self conjugacy under the operation of charge conjugation

$$
S_{[1 / 2]}^{c} \lambda\left(p^{\mu}\right)= \pm \lambda\left(p^{\mu}\right), \quad S_{[1 / 2]}^{c} \rho\left(p^{\mu}\right)= \pm \rho\left(p^{\mu}\right)
$$

determines $\zeta_{\lambda}^{S}=+i=\zeta_{\rho}^{S}$ for the self charge conjugate spinors $\lambda^{S}\left(p^{\mu}\right)$ and $\rho^{S}\left(p^{\mu}\right)$; and $\zeta_{\lambda}^{A}=$ $-i=\zeta_{\rho}^{A}$ for the anti-self charge conjugate spinors $\lambda^{A}\left(p^{\mu}\right)$ and $\rho^{A}\left(p^{\mu}\right)$. The $\lambda^{S}\left(p^{\mu}\right)$ are thus seen to coincide with the McLennan-Case construct [9,10].

For spin-1, on the other hand, the requirement of self/anti-self charge conjugacy cannot be satisfied. That is, there does not exist a $\zeta$ that can satisfy the spin-1 counterpart of the requirement (10). We find, however, that the requirement of self/anti-self conjugacy under charge conjugation can be replaced by the requirement of self/anti-self conjugacy under the operation of $\Gamma^{5} S_{[1]}^{c}$, where $\Gamma^{5}$ is the chirality operator for the $(1,0) \oplus(0,1)$ representation space and reads: $\square$

\footnotetext{
${ }^{7}$ All explicit expressions for the $2(2 j+1) \times 2(2 j+1)$ matrices, $\Gamma$, that appear in this paper are given in the generalized chiral/Weyl (W) representation. These matrices are related to the generalized canonical/Dirac (D) representation, which for spin-1/2 reduces to the familiar representation of Bjorken and Drell's text [23], via the following expression
} 


$$
\Gamma^{5}=\left(\begin{array}{cc}
\mathbb{1}_{3} & 0 \\
0 & -\mathbb{1}_{3}
\end{array}\right)
$$

with similar expressions for other spins. The requirement

$$
\left[\Gamma^{5} S_{[1]}^{c}\right] \lambda\left(p^{\mu}\right)= \pm \lambda\left(p^{\mu}\right), \quad\left[\Gamma^{5} S_{[1]}^{c}\right] \rho\left(p^{\mu}\right)= \pm \rho\left(p^{\mu}\right)
$$

determines $\zeta_{\lambda}^{S}=+1=\zeta_{\rho}^{S}$ for the self $\left[\Gamma^{5} S_{[1]}^{c}\right]$-conjugate spinors $\lambda^{S}\left(p^{\mu}\right)$ and $\rho^{S}\left(p^{\mu}\right)$; and $\zeta_{\lambda}^{A}=$ $-1=\zeta_{\rho}^{A}$ for the anti-self $\left[\Gamma^{5} S_{[1]}^{c}\right]$-conjugate spinors $\lambda^{A}\left(p^{\mu}\right)$ and $\rho^{A}\left(p^{\mu}\right)$.

For spin-1/2, the often repeated assertion that a Majorana spinor is a Weyl spinor in fourcomponent form is somewhat misleading as a simple counting of the degrees of freedom immediately reveals. For the massive case, there are four $\lambda\left(p^{\mu}\right)$-type spinors - two $\lambda^{S}\left(p^{\mu}\right)$ and two $\lambda^{S}\left(p^{\mu}\right)$. For comparison, the associated Weyl spinor, $\phi_{L}\left(p^{\mu}\right)$, has only two degrees of freedom. Same arguments apply to $\rho\left(p^{\mu}\right)$ and $\phi_{R}\left(p^{\mu}\right)$. It will be shown later that $\lambda^{S}\left(p^{\mu}\right)$ are positive energy solutions and $\lambda^{A}\left(p^{\mu}\right)$ are negative energy solutions of an appropriate wave equation.

\section{Helicity and Type-II Spinors}

For convenience, we define self/anti-self $\theta$-conjugacy to be self/anti-self conjugacy under the operation of charge conjugation for spin-1/2 and under the operation of $\left[\Gamma^{5} S_{[1]}^{c}\right]$ for spin-1. Let $\phi_{L, R}^{h^{\prime}}\left(p^{\mu}\right)$ be an eigenstate of the helicity operator

$$
\mathbf{J} \cdot \widehat{\mathbf{p}} \phi_{L, R}^{h^{\prime}}\left(p^{\mu}\right)=h^{\prime} \phi_{L, R}^{h^{\prime}}\left(p^{\mu}\right) \quad ;
$$

the Wigner-identity $\Theta_{[j]} \mathbf{J} \Theta_{[j]}^{-1}=-\mathbf{J}^{*}$ then implies that

$$
\mathbf{J} \cdot \widehat{\mathbf{p}} \Theta_{[j]}\left[\phi_{L, R}^{h^{\prime}}\left(p^{\mu}\right)\right]^{*}=-h^{\prime} \Theta_{[j]}\left[\phi_{L, R}^{h^{\prime}}\left(p^{\mu}\right)\right]^{*}
$$

That is, if $\phi_{L, R}^{h^{\prime}}\left(p^{\mu}\right)$ are eigenvectors of $\mathbf{J} \cdot \widehat{\mathbf{p}}$, then $\Theta_{[j]}\left[\phi_{L, R}^{h^{\prime}}\left(p^{\mu}\right)\right]^{*}$ are eigenvectors of $\mathbf{J} \cdot \widehat{\mathbf{p}}$ with opposite eigenvalues to those associated with $\phi_{L, R}^{h^{\prime}}\left(p^{\mu}\right)$. An inspection of (6) when coupled with this result implies that the self/anti-self $\theta$-conjugate spinors cannot be in helicity eigenstates. The helicity operator for the $(j, 0) \oplus(0, j)$ representation space is defined as

$$
h \equiv\left(\begin{array}{cc}
\mathbf{J} \cdot \widehat{\mathbf{p}} & 0 \\
0 & \mathbf{J} \cdot \widehat{\mathbf{p}}
\end{array}\right)
$$

Given $\phi_{L, R}^{h^{\prime}}\left(p^{\mu}\right)$ as eigenstates of $h$, the $\lambda\left(p^{\mu}\right)$ and $\rho\left(p^{\mu}\right)$ are readily seen to be eigenstates of the operator

$$
\eta \equiv-\Gamma^{5} h
$$

$$
\Gamma_{D}=S \Gamma_{W} S^{-1} \quad, \quad S=\frac{1}{\sqrt{2}}\left(\begin{array}{cc}
\mathbb{1} & \mathbb{1} \\
\mathbb{1} & -\mathbb{1}
\end{array}\right),
$$

with $\mathbb{1}=\mathrm{a}(2 j+1) \times(2 j+1)$ identity matrix. 
We shall call $\eta$ a chiral helicity operator and its eigenvalues chiral helicities.

Explicit Construction of $\lambda\left(p^{\mu}\right)$ for Spin-1/2 and Spin-1

The general form of the $(1 / 2,0) \oplus(0,1 / 2)$ and $(1,0) \oplus(0,1)$ representation-space $\lambda\left(p^{\mu}\right)$ rest spinors is obtained from (6) by setting $p^{\mu}=\grave{p}^{\mu}$ and equating $\zeta_{\lambda}$ in accordance with their values determined above. Once the rest spinors $\lambda\left({ }^{\mu} \mu\right)$ are written down, the $\lambda\left(p^{\mu}\right)$ follow by the application of an appropriate Wigner boost:

$$
\lambda\left(p^{\mu}\right)=W\left(j, p^{\mu} \leftarrow \stackrel{\circ}{p}^{\mu}\right) \lambda\left(\stackrel{\circ}{p}^{\mu}\right)
$$

where (Using Eq. (3) and appropriate expansions of $\exp ( \pm \mathbf{J} \cdot \varphi)$.) [

$$
\begin{aligned}
& W\left(1 / 2, p^{\mu} \leftarrow \stackrel{p}{ }^{\mu}\right)=\left(\frac{E+m}{2 m}\right)^{1 / 2}\left(\begin{array}{cc}
\mathbb{1}+(E+m)^{-1} \boldsymbol{\sigma} \cdot \boldsymbol{p} & 0 \\
0 & \mathbb{1}-(E+m)^{-1} \boldsymbol{\sigma} \cdot \boldsymbol{p}
\end{array}\right), \\
& W\left(1, p^{\mu} \leftarrow \grave{p}^{\mu}\right)= \\
& \left(\begin{array}{cc}
\mathbb{1}+m^{-1} \mathbf{J} \cdot \mathbf{p}+(m(E+m))^{-1}(\mathbf{J} \cdot \mathbf{p})^{2} & 0 \\
0 & \mathbb{1}-m^{-1} \mathbf{J} \cdot \mathbf{p}+(m(E+m))^{-1}(\mathbf{J} \cdot \mathbf{p})^{2}
\end{array}\right) .
\end{aligned}
$$

To construct $\lambda\left({ }_{p}^{\mu}\right)$ that are eigenstates of the chiral helicity operator, we choose $\phi_{L}\left(\stackrel{\circ}{p}^{\mu}\right)$ to be eigenstates of $\mathbf{J} \cdot \widehat{\mathbf{p}}$.

The explicit expressions for the $\lambda\left(p^{\mu}\right)$ using the above procedure, and exploiting definition (13) and identity (14), yields the following results:

I. For Spin- $1 / 2$

$$
\begin{aligned}
& \lambda_{\uparrow}^{S}\left(p^{\mu}\right)=\left(\frac{E+m}{2 m}\right)^{1 / 2}\left(\begin{array}{c}
\left\{1-(E+m)^{-1}|\mathbf{p}|\right\} i \Theta_{[1 / 2]}\left[\phi_{L}^{+1 / 2}\left(\stackrel{p}{\mu}^{\mu}\right)\right]^{*} \\
\left\{1-(E+m)^{-1}|\mathbf{p}|\right\} \phi_{L}^{+1 / 2}\left(\grave{p}^{\mu}\right)
\end{array}\right), \\
& \lambda_{\downarrow}^{S}\left(p^{\mu}\right)=\left(\frac{E+m}{2 m}\right)^{1 / 2}\left(\begin{array}{c}
\left\{1+(E+m)^{-1}|\mathbf{p}|\right\} i \Theta_{[1 / 2]}\left[\phi_{L}^{-1 / 2}\left(\stackrel{\circ}{p}^{\mu}\right)\right]^{*} \\
\left\{1+(E+m)^{-1}|\mathbf{p}|\right\} \phi_{\mathbf{L}}^{-1 / 2}\left(\stackrel{p}{p}^{\mu}\right)
\end{array}\right) .
\end{aligned}
$$

\section{For Spin-1}

$$
\begin{aligned}
& \lambda_{\uparrow}^{S}\left(p^{\mu}\right)=\left(\begin{array}{c}
\left\{\mathbb{1}-m^{-1}|\mathbf{p}|+(m(E+m))^{-1}|\mathbf{p}|^{2}\right\} \Theta_{[1]}\left[\phi_{L}^{+1}\left(\stackrel{\circ}{p}^{\mu}\right)\right]^{*} \\
\left\{\mathbb{1}-m^{-1}|\mathbf{p}|+(m(E+m))^{-1}|\mathbf{p}|^{2}\right\} \phi_{L}^{+1}\left(\stackrel{\circ}{p}^{\mu}\right)
\end{array}\right), \\
& \lambda_{\rightarrow}^{S}\left(p^{\mu}\right)=\left(\begin{array}{c}
\Theta_{[1]}\left[\phi_{L}^{0}\left(\stackrel{\circ}{p}^{\mu}\right)\right]^{*} \\
\phi_{L}^{0}\left(\stackrel{o}{p}^{\mu}\right)
\end{array}\right), \\
& \lambda_{\downarrow}^{S}\left(p^{\mu}\right)=\left(\begin{array}{c}
\left\{\mathbb{1}+m^{-1}|\mathbf{p}|+(m(E+m))^{-1}|\mathbf{p}|^{2}\right\} \Theta_{[1]}\left[\phi_{L}^{-1}\left(\stackrel{\circ}{p}^{\mu}\right)\right]^{*} \\
\left\{\mathbb{1}+m^{-1}|\mathbf{p}|+(m(E+m))^{-1}|\mathbf{p}|^{2}\right\} \phi_{L}^{-1}\left(\check{p}^{\mu}\right)
\end{array}\right) .
\end{aligned}
$$

\footnotetext{
${ }^{8}$ It is a noteworthy observation that the helicity operator $\mathbf{J} \cdot \widehat{\mathbf{p}}$ enters the Wigner boosts (18) and (19) in a non-trivial fashion. The linearity of the spin- $1 / 2$ Dirac equation in $\partial_{\mu}$, and the quadratic nature of the modified Weinberg equation [16,3], lie in this observation. The specific difference arises as a result of $j$-dependent behavior of $(\mathbf{J} \cdot \mathbf{p})^{n} ; n=$ integer.
} 
For spin-1/2, the subscripts $\uparrow$ and $\downarrow$ correspond to $\lambda\left(\stackrel{\circ}{p}^{\mu}\right)$ constructed out of $\phi_{L}^{+}\left(\stackrel{\circ}{p}^{\mu}\right)$ and $\phi_{L}^{-}\left(\stackrel{\circ}{p}^{\mu}\right)$, respectively, and are to be interpreted as chiral helicities. Similarly for spin-1, the subscripts $\uparrow, \rightarrow$, and $\downarrow$ correspond to $\lambda\left(\stackrel{\circ}{p}^{\mu}\right)$ constructed out of $\phi_{L}^{+1}\left(\stackrel{\circ}{p}^{\mu}\right), \phi_{L}^{0}\left(\stackrel{\circ}{p}^{\mu}\right)$, and $\phi_{L}^{-1}\left(\stackrel{\circ}{p}^{\mu}\right)$, respectively.

The expressions for the anti-self charge-conjugate spinors $\lambda^{A}\left(p^{\mu}\right)$ are obtained by replacing $i \Theta_{[1 / 2]}$ by $-i \Theta_{[1 / 2]}$, for spin-1/2, and $\Theta_{[1]}$ by $-\Theta_{[1]}$, for spin-1, in the above expressions and at the same time replacing $\lambda^{S}\left(p^{\mu}\right)$ by $\lambda^{A}\left(p^{\mu}\right)$ without changing the chiral helicity indices.

If we restrict ourselves to the physically acceptable norms, $N^{2}$, for $\phi^{ \pm}\left(p^{\mu}\right)$ such that for massless particles, all rest-spinors vanish (because massless particles cannot be at rest); then, first considering spin-1/2, an inspection of $\lambda\left(p^{\mu}\right)$ given by Eqs. (20a) and (20b) immediately reveals that for massless particles there exists a kinematical asymmetry for the self/anti-self charge-conjugate spinors in the $(1 / 2,0) \oplus(0,1 / 2)$ representation space. For massless particles, $\lambda_{\uparrow}^{S}\left(p^{\mu}\right)\left(\right.$ and $\left.\lambda_{\uparrow}^{A}\left(p^{\mu}\right)\right)$ identically vanish. However, this vanishing should not be associated with the norm we have chosen. The norm simply avoids the unphysical and singular norm of the massless spinors. The physical origin of this asymmetry lies in the fact that $\left\{\mathbb{1}+(E+m)^{-1} \boldsymbol{\sigma} \cdot \boldsymbol{p}\right\}$, which appears in the Wigner boost (18), acting on $\Theta_{[1 / 2]}\left[\phi_{L}^{+}\left(\stackrel{\circ}{p}^{\mu}\right)\right]^{*}$, a factor that originates from the requirement of self/anti-self charge conjugacy, on exploiting the identity (14), which has its origin in the very specific property of the Wigner's operator $\left(\Theta_{[j]} \mathbf{J} \Theta_{[j]}^{-1}=-\mathbf{J}^{*}\right)$, conspire to yield $\left\{\mathbb{1}-(E+m)^{-1}|\mathbf{p}|\right\} \Theta_{[1 / 2]}\left[\phi_{L}^{+}\left(\stackrel{\circ}{p}^{\mu}\right)\right]^{*}$, which for the massless case vanishes. Similar remarks also apply to spin-1.

The most general forms for the $\phi^{h^{\prime}}\left(\stackrel{\circ}{p}^{\mu}\right)$ that appear in expressions (20a) to (21d) are:

A. For Spin-1/2

$$
\begin{aligned}
& \phi_{L}^{+1 / 2}\left(\stackrel{\circ}{p}^{\mu}\right)=N e^{i \vartheta_{1}}\left(\begin{array}{c}
\cos (\theta / 2) e^{-i \phi / 2} \\
\sin (\theta / 2) e^{i \phi / 2}
\end{array}\right), \\
& \phi_{L}^{-1 / 2}\left(\stackrel{\circ}{p}^{\mu}\right)=N e^{i \vartheta_{2}}\left(\begin{array}{c}
\sin (\theta / 2) e^{-i \phi / 2} \\
-\cos (\theta / 2) e^{i \phi / 2}
\end{array}\right) .
\end{aligned}
$$

\section{B. For Spin-1}

$$
\begin{aligned}
& \phi_{L}^{+1}\left(\stackrel{\circ}{p}^{\mu}\right)=\mathrm{N} e^{i \delta_{1}}\left(\begin{array}{l}
\frac{1}{2}(1+\cos \theta) e^{-i \phi} \\
\sqrt{\frac{1}{2}} \sin \theta \\
\frac{1}{2}(1-\cos \theta) e^{i \phi}
\end{array}\right) \\
& \phi_{L}^{0}\left(\stackrel{\circ}{p}^{\mu}\right)=\mathrm{N} e^{i \delta_{2}}\left(\begin{array}{l}
-\sqrt{\frac{1}{2}} \sin \theta e^{-i \phi} \\
\cos \theta \\
\sqrt{\frac{1}{2}} \sin \theta e^{i \phi}
\end{array}\right) \\
& \phi_{L}^{-1}\left(\stackrel{p}{p}^{\mu}\right)=\mathrm{N} e^{i \delta_{3}}\left(\begin{array}{l}
\frac{1}{2}(1-\cos \theta) e^{-i \phi} \\
-\sqrt{\frac{1}{2}} \sin \theta \\
\frac{1}{2}(1+\cos \theta) e^{i \phi}
\end{array}\right)
\end{aligned}
$$

Here, $\theta$ and $\phi$ are the standard polar and azimuthal angles associated with $\mathbf{p}$.

\footnotetext{
${ }^{9}$ A convenient choice [3,22] satisfying this requirement is $N=m^{j} \times$ (phase factor).
} 
Since both $\phi_{L}\left(\hat{p}^{\mu}\right)$ and its complex conjugate $\phi_{L}^{*}\left(\grave{p}^{\mu}\right)$ enter a given $\lambda\left(p^{\mu}\right)$, the covariant norm $\bar{\lambda}\left(p^{\mu}\right) \lambda\left(p^{\mu}\right)$ depends on the choice of phase factors $e^{\vartheta_{1,2}}$ and $e^{\delta_{1,2,3}}$. This is manifest in Tables I and II, where $\bar{\lambda}\left(p^{\mu}\right) \lambda\left(p^{\mu}\right)$ are explicitly tabulated. Any phase choice for which $\vartheta_{1}+\vartheta_{2}$ vanishes yields a bi-orthonormal set for spin-1/2. Similarly, for spin-1, phase choices for which $\delta_{3}+\delta_{1}$ and $\delta_{2}$ vanish yield a bi-orthonormal set. We shall make these choices in the rest of this paper. In fact, for convenience, we set $\vartheta_{1}=0=\vartheta_{2}$ for spin- $1 / 2$; and $\delta_{1}=\delta_{2}=0=\delta_{3}$ for spin- 1 . For spin-1, as well as for spin-1/2, $\lambda^{S}\left(p^{\mu}\right)$ and $\lambda^{A}\left(p^{\mu}\right)$ span mutually orthogonal sub-spaces, with the completeness relations given by:

$$
\begin{aligned}
\frac{-1}{2 i N^{2}}\left[\left\{\lambda_{\uparrow}^{S}\left(p^{\mu}\right) \bar{\lambda}_{\downarrow}^{S}\left(p^{\mu}\right)-\right.\right. & \left.\lambda_{\downarrow}^{S}\left(p^{\mu}\right) \bar{\lambda}_{\uparrow}^{S}\left(p^{\mu}\right)\right\}- \\
& \left.\left\{\lambda_{\uparrow}^{A}\left(p^{\mu}\right) \bar{\lambda}_{\downarrow}^{A}\left(p^{\mu}\right)-\lambda_{\downarrow}^{A}\left(p^{\mu}\right) \bar{\lambda}_{\uparrow}^{A}\left(p^{\mu}\right)\right\}\right]=\mathbb{1},
\end{aligned}
$$

for spin- $1 / 2$; and for spin-1 by

$$
\begin{aligned}
\frac{1}{2 \mathrm{~N}^{2}}\left[\left\{\lambda_{\uparrow}^{S}\left(p^{\mu}\right) \bar{\lambda}_{\downarrow}^{S}\left(p^{\mu}\right)-\right.\right. & \left.\lambda_{\rightarrow}^{S}\left(p^{\mu}\right) \bar{\lambda}_{\rightarrow}^{S}\left(p^{\mu}\right)+\lambda_{\downarrow}^{S}\left(p^{\mu}\right) \bar{\lambda}_{\uparrow}^{S}\left(p^{\mu}\right)\right\}- \\
& \left.\left\{\lambda_{\uparrow}^{A}\left(p^{\mu}\right) \bar{\lambda}_{\downarrow}^{A}\left(p^{\mu}\right)-\lambda_{\rightarrow}^{A}\left(p^{\mu}\right) \bar{\lambda}_{\rightarrow}^{A}\left(p^{\mu}\right)+\lambda_{\downarrow}^{A}\left(p^{\mu}\right) \bar{\lambda}_{\uparrow}^{A}\left(p^{\mu}\right)\right\}\right]=\mathbb{1} .
\end{aligned}
$$

The construction of the $\rho\left(p^{\mu}\right)$ spinors follows in a parallel fashion.

New Wave Equations for Spin-1/2 and Spin-1: Wave Equation for $\lambda\left(p^{\mu}\right)$

To obtain a wave equation satisfied by $\lambda\left(p^{\mu}\right)$ we must first generalize the Ryder-Burgard relation. This is accomplished be defining a $(2 j+1) \times(2 j+1)$ matrix $\Xi_{[j]}$ such that

$$
\left[\phi_{L}^{h^{\prime}}\left(\stackrel{\circ}{p}^{\mu}\right)\right]^{*}=\Xi_{[j]} \phi_{L}^{h^{\prime}}\left(\stackrel{\circ}{p}^{\mu}\right)
$$

With the choice of phases $e^{i \vartheta}$ and $e^{i \delta}$ introduced above, $\Xi_{[j]}$ for spin- $1 / 2$ and spin-1 read:

$$
\Xi_{[1 / 2]}=\left(\begin{array}{cc}
e^{i \phi} & 0 \\
0 & e^{-i \phi}
\end{array}\right) \quad, \quad \Xi_{[1]}=\left(\begin{array}{ccc}
e^{i 2 \phi} & 0 & 0 \\
0 & 1 & 0 \\
0 & 0 & e^{-i 2 \phi}
\end{array}\right) .
$$

Next, for convenience, we introduce the abbreviation

$$
\chi_{R}\left(p^{\mu}\right) \equiv\left(\zeta_{\lambda} \Theta_{[j]}\right) \phi_{L}^{*}\left(p^{\mu}\right)
$$

\footnotetext{
${ }^{10}$ Recall that $\bar{\psi}\left(p^{\mu}\right)$ in the $(j, 0) \oplus(0, j)$ representation space is defined as $\psi^{\dagger}\left(p^{\mu}\right) \times\left(\begin{array}{cc}0 & \mathbb{1} \\ \mathbb{1} & 0\end{array}\right)$; where $\mathbb{1}=\mathrm{a}(2 j+1) \times(2 j+1)$ identity matrix.

11 Cf. Equation (26) with the equation in the second line on p. 44 of Ryder's book [2]. The corrected form of the indicated equation of Ryder was obtained in discussions with Burgard 24] and can be found in Ref. [3]. In part, the analysis of Ref. [3] yields Dirac-like modified Weinberg wave equations for $(j, 0) \oplus(0, j)$ spinors.
} 
and couple the transformation properties

$$
\begin{aligned}
& \chi_{R}\left(p^{\mu}\right)=\exp (\mathbf{J} \cdot \boldsymbol{\varphi}) \chi_{R}\left(\stackrel{\circ}{p}^{\mu}\right), \\
& \phi_{L}\left(p^{\mu}\right)=\exp (-\mathbf{J} \cdot \boldsymbol{\varphi}) \phi_{L}\left(\stackrel{\circ}{p}^{\mu}\right),
\end{aligned}
$$

with (26) to obtain (after a short string of algebraic manipulations that exploit some already indicated properties of $\Theta_{[j]}$ and $\zeta_{\lambda}$ and are similar in nature to those found in Sec. 2.3 of Ryder's book [2] and Refs. [3:4])

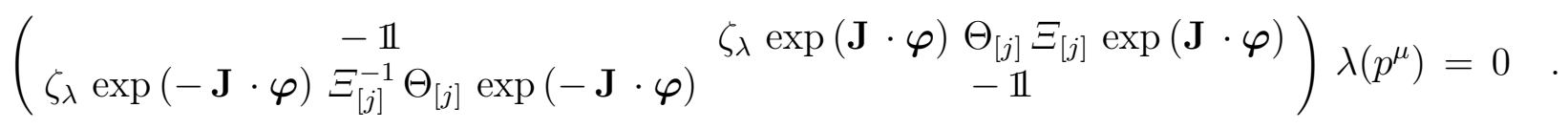

This is the general wave equation satisfied by arbitrary-spin $\lambda\left(p^{\mu}\right)$.

For spin-1/2 the equation (30) takes the form

$$
\left(\begin{array}{cc}
-2 m(E+m) \mathbb{1} & \zeta_{\lambda}\{(E+m) \mathbb{1}+\boldsymbol{\sigma} \cdot \mathbf{p}\} \Theta_{[1 / 2]} \Xi_{[1 / 2]} \\
\zeta_{\lambda}\{(E+m) \mathbb{1}-\boldsymbol{\sigma} \cdot \mathbf{p}\} & \Xi_{[1 / 2]}^{-1} \Theta_{[1 / 2]} \\
\times\{(E+m) \mathbb{1}-\boldsymbol{\sigma} \cdot \mathbf{p}\} & \times\{(E+m) \mathbb{1}+\boldsymbol{\sigma} \cdot \mathbf{p}\}
\end{array}\right) \lambda\left(p^{\mu}\right)=0
$$

and for spin-1 it becomes

$$
\left(\begin{array}{cc}
-\{m(m+E)\}^{2} \mathbb{1} & \zeta_{\lambda}\left\{\cdots A_{+} \cdots\right\} \Theta_{[1]} \Xi_{[1]}\left\{\cdots A_{+} \cdots\right\} \\
\zeta_{\lambda}\left\{\cdots A_{-} \cdots\right\} \Xi_{[1]}^{-1} \Theta_{[1]}\left\{\cdots A_{-} \cdots\right\} & -\{m(m+E)\}^{2} \mathbb{1}
\end{array}\right) \lambda\left(p^{\mu}\right)=0
$$

with

$$
\left\{\cdots A_{ \pm} \cdots\right\}=\left\{\left(\eta_{\mu \nu} p^{\mu} p^{\nu}+m E\right) \mathbb{1} \pm(m+E) \mathbf{J} \cdot \mathbf{p}+(\mathbf{J} \cdot \mathbf{p})^{2}\right\}
$$

and $\eta_{\mu \nu}$ is the flat space-time metric with $\operatorname{diag}(1,-1,-1,-1)$.

Both for spin-1/2 and spin-1, using the symbolic manipulation program DOEMACSYMA, we find that while $\lambda^{S}\left(p^{\mu}\right)$ are the positive energy solutions with $E=+\sqrt{m^{2}+\mathbf{p}^{2}}, \lambda^{A}\left(p^{\mu}\right)$ are the negative energy solutions with $E=-\sqrt{m^{2}+\mathbf{p}^{2}}$. These results suggest a particle-antiparticle interpretation. Since the particle-hole picture so useful for fermions cannot be generalized to bosons (because filling the negative energy sea works for fermions but not for bosons), we shall, following Hatfield [26], interpret negative energy particles (fermions or bosons) propagating backward in time as antiparticles in accordance with the Stükelberg/Feynman-Wheeler [27 picture of space-time. Similar results are obtained for $\rho\left(p^{\mu}\right)$.

For type II spinors, it should be explicitly noted that, unlike the case for type I spinors, the Charge Conjugation operator does not take the positive (negative) energy solutions into negative (positive) energy solutions.

Before proceeding further, we make a few brief remarks on the existence of more than one equation (unconnected by unitary transformations) in a given representation space. As early as 1932 Majorana [8] obtained a relativistic wave equation for spin-1/2 particles (which included a tower of higher spins also) starting from the demand that all its solutions have positive energy. Almost four decades later in 1971 Dirac [28] proposed a relativistic wave equation for bosons, which again allowed only positive-energy solutions and had the property that it described particles that did 
not interact electromagnetically (see Dirac's comments on pp. 68-69 in Ref. [29]). The reason that we obtain an additional wave equation in the $(1 / 2,0) \oplus(0,1 / 2)$ representation space that is different from Dirac's famous equation of 1928 and a new wave equation in the $(1,0) \oplus(0,1)$ representation space that is different from (the modified [3]) Weinberg's wave equation [16, 30 of 1964 is that wave equations are determined by the (physically-motivated) constraints that we impose on its solutions and transformation properties of the objects in the representation space under consideration. In general, different constraints yield different wave equations. Dirac (1971,1972) and Majorana (1932) imposed the constraint of positive-energy solutions. For the solutions we consider, we imposed the requirement of the self/anti-self charge conjugacy for spin-1/2 and self/anti-self conjugacy under the operation of $\Gamma^{5} S_{[1]}^{c}$ for spin-1. Dirac's famous equation of 1928 describes eigenspinors of the Charge operator.

\section{Some Considerations on Parity}

We begin with the study of parity covariance of equations (31) and (32). Define the operator that acts on $\lambda\left(p^{\mu}\right)$ in the equation (31) as $\mathcal{O}_{[1 / 2]}\left(p^{\mu}\right)$. Similarly, referring to (32), introduce $\mathcal{O}_{[1]}\left(p^{\mu}\right)$. The parity covariance of equations $\mathcal{O}_{[1 / 2]}\left(p^{\mu}\right) \lambda\left(p^{\mu}\right)=0$ and $\mathcal{O}_{[1]}\left(p^{\mu}\right) \lambda\left(p^{\mu}\right)=0$ demands that we seek operators $\mathcal{S}_{[\alpha]}^{s}$ such that under $\mathcal{P}$

$$
\begin{aligned}
\mathcal{O}_{[\alpha]}\left(p^{\mu}\right) & \rightarrow \mathcal{O}_{[\alpha]}^{\prime}\left(p^{\mu}\right)=\mathcal{S}_{[\alpha]}^{s} \mathcal{O}_{[\alpha]}\left(p^{\mu}\right)\left[\mathcal{S}_{[\alpha]}^{s}\right]^{-1} \\
\lambda\left(p^{\mu}\right) & \rightarrow \lambda^{\prime}\left(p^{\mu}\right)=\mathcal{S}_{[\alpha]}^{s} \lambda\left(p^{\mu}\right) \quad \alpha=1 / 2,1
\end{aligned}
$$

with $\mathcal{O}_{[\alpha]}^{\prime}\left(p^{\mu}\right)=\mathcal{O}_{[\alpha]}\left(p^{\prime \mu}\right)$ and $\lambda^{\prime}\left(p^{\mu}\right)=\lambda\left(p^{\prime \mu}\right)$. Here, $p^{\prime \mu}$ is the parity-transformed $p^{\mu}$ and reads $(E,-\mathbf{p})$ for $p^{\mu}=(E, \mathbf{p})$. On exploiting the fact that $\Theta_{[\alpha]} \Xi_{[\alpha]}=\Xi_{[\alpha]}^{-1} \Theta_{[\alpha]}$, such operators are found to be identical to those already given in (7) and (8); that is: $\mathcal{S}_{[\alpha]}^{s}=S_{[\alpha]}^{s}$. This is not surprising. Just as the operator of parity in the $(j, 0) \oplus(0, j)$ representation space is independent of which wave equation is under study, similarly the operations of charge conjugation and time reversal [7] do not depend on a specific wave equation. Within the context of the logical framework of the present paper, without this being true we would not even know how to define self-/anti self conjugate $(j, 0) \oplus(0, j)$ spinors. These remarks should not be interpreted to mean that there may not arise certain subtle differences between the operations of $\mathrm{P}$ and $\mathrm{C}$ in the Fock space [19].

We no longer pursue the subject of CPT covariance any further. A detailed analysis of these operators, within the context of a Foldy-Nigam-Bargmann-Wightman-Wigner type quantum field theory, was recently published in Ref. [3]. To incorporate the Nigam and Foldy considerations in the work of Ref. [3] one simply follows the details of [19] in a straightforward manner.

12 The charge conjugation and parity operations in the $(j, 0) \oplus(0, j)$ representation space for spin$1 / 2$ and spin-1 are given by (7) and (8). These are supplemented by the time-reversal operators [20, 3] (within a global phase factor)

$$
S_{[1 / 2]}(T)=-\left(\begin{array}{cc}
0 & \Theta_{[1 / 2]} \\
\Theta_{[1 / 2]} & 0
\end{array}\right) \quad, \quad S_{[1]}(T)=\left(\begin{array}{cc}
\Theta_{[1]} & 0 \\
0 & -\Theta_{[1]}
\end{array}\right) .
$$

However, it should be again recalled that the analysis by Nigam and Foldy reveals that the Parity operator in the Fock space depends on the Charge operator. 
With the choice of phases $e^{i \vartheta}$ and $e^{i \delta}$ made above, the effect of the parity operator, $S_{[j]}^{s}$, on $\lambda^{S}\left(p^{\mu}\right)$ and $\lambda^{A}\left(p^{\mu}\right)$ spinors is given as follows:

a. For Spin-1/2

$$
\begin{array}{ll}
\gamma^{0} \lambda_{\uparrow}^{S}\left(p^{\mu}\right)=-i \lambda_{\downarrow}^{S}\left(p^{\prime \mu}\right), & \gamma^{0} \lambda_{\downarrow}^{S}\left(p^{\mu}\right)=+i \lambda_{\uparrow}^{S}\left(p^{\prime \mu}\right) \\
\gamma^{0} \lambda_{\uparrow}^{A}\left(p^{\mu}\right)=+i \lambda_{\downarrow}^{A}\left(p^{\prime \mu}\right), & \gamma^{0} \lambda_{\downarrow}^{A}\left(p^{\mu}\right)=-i \lambda_{\uparrow}^{A}\left(p^{\prime \mu}\right)
\end{array}
$$

\section{For Spin-1}

$$
\begin{array}{ll}
\gamma_{00} \lambda_{\uparrow}^{S}\left(p^{\mu}\right)=+\lambda_{\downarrow}^{S}\left(p^{\prime \mu}\right), & \gamma_{00} \lambda_{\rightarrow}^{S}\left(p^{\mu}\right)=-\lambda_{\rightarrow}^{S}\left(p^{\prime \mu}\right), \quad \gamma_{00} \lambda_{\downarrow}^{S}\left(p^{\mu}\right)=+\lambda_{\uparrow}^{S}\left(p^{\prime \mu}\right), \\
\gamma_{00} \lambda_{\uparrow}^{A}\left(p^{\mu}\right)=-\lambda_{\downarrow}^{A}\left(p^{\prime \mu}\right), & \gamma_{00} \lambda_{\rightarrow}^{A}\left(p^{\mu}\right)=+\lambda_{\rightarrow}^{A}\left(p^{\prime \mu}\right), \quad \gamma_{00} \lambda_{\downarrow}^{A}\left(p^{\mu}\right)=-\lambda_{\uparrow}^{A}\left(p^{\prime \mu}\right) .
\end{array}
$$

The $\lambda\left(p^{\mu}\right)$ for spin-1/2 are not eigenspinors of the parity operator. This is not related to the fact that $S_{[1 / 2]}(\mathcal{C})$ and $S_{[1 / 2]}(\mathcal{P})$ do not commute. Since $S_{[1 / 2]}(\mathcal{C})$ is not linear, it is possible to have a simultaneous set of eigenspinors for $S_{[1 / 2]}(\mathcal{C})$ and $S_{[1 / 2]}(\mathcal{P})$; but such a set does not have its eigenspinors of type II. [13]

The $\lambda\left(p^{\mu}\right)$ for spin-1 can however be made into eigenspinors of the Parity operator (without destroying the $\theta$-conjugacy). Specifically,

$$
\lambda_{ \pm}^{S}\left(p^{\mu}\right) \equiv \frac{1}{\sqrt{2}}\left[\lambda_{\uparrow}^{S}\left(p^{\mu}\right) \pm \lambda_{\downarrow}^{S}\left(p^{\mu}\right)\right], \quad \lambda_{0}\left(p^{\mu}\right) \equiv \lambda_{\rightarrow}^{S}\left(p^{\mu}\right)
$$

[with a similar expressions for the $\left.\lambda_{ \pm, 0}^{A}\left(p^{\mu}\right)\right]$ are simultaneously eigenspinors of the $\Gamma^{5} S_{[1]}(\mathcal{C})$ and $S_{[1]}(\mathcal{P})$ operators:

$$
\begin{aligned}
& \Gamma^{5} S_{[1]}(\mathcal{C}) \lambda_{ \pm, 0}^{S}\left(p^{\mu}\right)=\lambda_{ \pm, 0}^{S}\left(p^{\mu}\right) \\
& \gamma_{00} \lambda_{ \pm}^{S}\left(p^{\mu}\right)=+\lambda_{ \pm}^{S}\left(p^{\prime \mu}\right), \quad \gamma_{00} \lambda_{0}^{S}\left(p^{\mu}\right)=-\lambda_{0}^{S}\left(p^{\prime \mu}\right)
\end{aligned}
$$

The subscript \pm no longer corresponds to the chiral helicity. $\lambda_{ \pm}^{S}\left(p^{\mu}\right)$ and $\lambda_{0}^{S}\left(p^{\mu}\right)$ carry opposite relative intrinsic parities [with similar statement being true for $\lambda_{ \pm, 0}^{A}\left(p^{\mu}\right)$ ]. If $\lambda_{ \pm, 0}\left(p^{\mu}\right)$ were realized as physical degrees of freedom then any interaction that induces transitions between the \pm and 0 degree of freedom would necessarily violate parity, except in the massless case where transitions to the 0 degree of freedom would be expected to vanish identically on the basis of arguments presented in the section entitled "Explicit Construction of $\lambda\left(p^{\mu}\right)$ for Spin-1/2 and Spin-1."

\section{Dirac-Like and Majorana-Like Fields}

On setting $\vartheta_{1}=0=\vartheta_{2}$ for spin- $1 / 2$, and $\delta_{1}=\delta_{2}=0=\delta_{3}$ for spin- 1 , as discussed before, the $\lambda\left(p^{\mu}\right)$ form a bi-orthogonal set of self/anti-self charge conjugate spinors. Similar results hold true for $\rho^{S}\left(p^{\mu}\right)$ and $\rho^{A}\left(p^{\mu}\right)$. In addition, there exist several identities that relate $\lambda^{S}\left(p^{\mu}\right)$ and $\rho^{A}\left(p^{\mu}\right)$, etc. (see, e.g., Eqs. (48a) and (48b) below).

\footnotetext{
${ }^{13}$ This argument was constructed with Christoph Burgard and George Kahrimanis. I thank them both.
} 
Since the set of self/anti-self charge conjugate spinors cannot be made orthonormal without destroying self/anti-self charge conjugacy, the noted bi-orthonormality suggests that the physical states be normed as:

$$
\beta^{\prime}\left\langle p^{\prime \mu}, \eta^{\prime} \mid p^{\mu}, \eta\right\rangle^{\beta}=(2 \pi)^{3} 2 p_{0} \delta^{3}\left(\mathbf{p}-\mathbf{p}^{\prime}\right) \delta_{\eta,-\eta^{\prime}} \delta_{\beta \beta^{\prime}} .
$$

Notational Note: $\pm \uparrow=\mp \downarrow$ and $-\rightarrow=\rightarrow ; \beta$ and $\beta^{\prime}$ can take values $S$ and $A$, which refer to the self and anti-self charge conjugacy identifying indices. Consider $\beta=\beta^{\prime}=S$, first. The lhs of (41) can now be written as

$$
\left\langle\operatorname{vac}\left|\left[a_{\eta^{\prime}}\left(p^{\prime \mu}\right), a_{\eta}^{\dagger}\left(p^{\mu}\right)\right]_{ \pm}\right| \operatorname{vac}\right\rangle \mp\left\langle\operatorname{vac}\left|a_{\eta}^{\dagger}\left(p^{\mu}\right) a_{\eta^{\prime}}\left(p^{\prime \mu}\right)\right| \operatorname{vac}\right\rangle=(2 \pi)^{3} 2 p_{0} \delta^{3}\left(\mathbf{p}-\mathbf{p}^{\prime}\right) \delta_{\eta,-\eta^{\prime}}
$$

where $\mid$ vac $\rangle$ represents the vacuum state. The second term in the lhs of the above equation vanishes identically, yielding

$$
\left[a_{\eta^{\prime}}\left(p^{\prime \mu}\right), a_{\eta}^{\dagger}\left(p^{\mu}\right)\right]_{ \pm}=(2 \pi)^{3} 2 p_{0} \delta^{3}\left(\mathbf{p}-\mathbf{p}^{\prime}\right) \delta_{\eta,-\eta^{\prime}} .
$$

Next we consider $\beta=\beta^{\prime}=A$, and obtain

$$
\left[b_{\eta^{\prime}}\left(p^{\prime \mu}\right), b_{\eta}^{\dagger}\left(p^{\mu}\right)\right]_{ \pm}=(2 \pi)^{3} 2 p_{0} \delta^{3}\left(\mathbf{p}-\mathbf{p}^{\prime}\right) \delta_{\eta,-\eta^{\prime}} .
$$

Finally, by considering $\beta=S$ and $\beta^{\prime}=A$ ( or, $\beta=A$ and $\beta^{\prime}=S$ ), we obtain

$$
\left[a_{\eta^{\prime}}\left(p^{\prime \mu}\right), b_{\eta}^{\dagger}\left(p^{\mu}\right)\right]_{ \pm}=0
$$

Using any set of two of the $\lambda^{S}\left(p^{\mu}\right), \lambda^{A}\left(p^{\mu}\right), \rho^{S}\left(p^{\mu}\right)$, and $\rho^{A}\left(p^{\mu}\right)$ that forms a complete set we can now introduce the quantum field motivated by reasons surrounding Eq. (9). Two examples follow (in the phenomenological context one must remain open to other inherent possibilities in the formalism):

1. A Dirac-like field

$$
\nu^{D L}(x) \equiv \int \frac{d^{3} \boldsymbol{p}}{(2 \pi)^{3}} \frac{1}{2 p_{0}} \sum_{\eta}\left[\lambda_{\eta}^{S}\left(p^{\mu}\right) a_{\eta}\left(p^{\mu}\right) \exp (-i p \cdot x)+\lambda_{\eta}^{A}\left(p^{\mu}\right) b_{\eta}^{\dagger}\left(p^{\mu}\right) \exp (+i p \cdot x)\right],
$$

or, on identifying $b_{\eta}^{\dagger}\left(p^{\mu}\right)$ with $a_{\eta}^{\dagger}\left(p^{\mu}\right)$ (in analogy with obtaining the Majorana field from the Dirac field)

2. A Majorana-like field

$$
\nu^{M L}(x) \equiv \int \frac{d^{3} \boldsymbol{p}}{(2 \pi)^{3}} \frac{1}{2 p_{0}} \sum_{\eta}\left[\lambda_{\eta}^{S}\left(p^{\mu}\right) a_{\eta}\left(p^{\mu}\right) \exp (-i p \cdot x)+\lambda_{\eta}^{A}\left(p^{\mu}\right) a_{\eta}^{\dagger}\left(p^{\mu}\right) \exp (+i p \cdot x)\right] .
$$


Note that neither $\nu^{D L}(x)$ nor $\nu^{M L}(x)$ is a self/anti-self $\theta$-conjugate field. However, both fields describe self/anti-self $\theta$-conjugate states and use of one or the other field leads to a set of phenomenon that does not have a complete overlap.

In reference to the parenthetic remark bracketed between Eqs. (45) and (46), we note the identities (for spin-1/2, similar identities exist for spin-1)

$$
\begin{array}{ll}
\rho_{\uparrow}^{S}\left(p^{\mu}\right)=-i \lambda_{\downarrow}^{A}\left(p^{\mu}\right), & \rho_{\downarrow}^{S}\left(p^{\mu}\right)=+i \lambda_{\uparrow}^{A}\left(p^{\mu}\right), \\
\rho_{\uparrow}^{A}\left(p^{\mu}\right)=+i \lambda_{\downarrow}^{S}\left(p^{\mu}\right), & \rho_{\downarrow}^{A}\left(p^{\mu}\right)=-i \lambda_{\uparrow}^{S}\left(p^{\mu}\right),
\end{array}
$$

These identities may be used to incorporate the left- and right-handed chiral helicities in the same spin-1/2 field. For instance, for this example case, we re-write $\nu^{D L}(x)$ as

$$
\nu_{2}^{D L}(x) \equiv \int \frac{d^{3} \boldsymbol{p}}{(2 \pi)^{3}} \frac{1}{2 p_{0}} \sum_{\eta}\left[\lambda_{\eta}^{S}\left(p^{\mu}\right) c_{\eta}\left(p^{\mu}\right) \exp (-i p \cdot x)+\rho_{\eta}^{S}\left(p^{\mu}\right) d_{\eta}^{\dagger}\left(p^{\mu}\right) \exp (+i p \cdot x)\right] .
$$

The $c_{\eta}\left(p^{\mu}\right)$ and $d_{\eta}^{\dagger}\left(p^{\mu}\right)$ differ from $a_{\eta}\left(p^{\mu}\right)$ and $b_{\eta}^{\dagger}\left(p^{\mu}\right)$ with appropriate phase factors dictated by Eqs. (48a) and (48b).

As shown already, in the massless limit, since $\lambda_{\downarrow}\left(p^{\mu}\right)$ and $\rho_{\uparrow}\left(p^{\mu}\right)$ are the only surviving spinors the massless spin-1/2 field $\nu_{2}^{D L}(x)$ contains only the following states: self-charge conjugate states $\left|p^{\mu}, \downarrow\right\rangle^{S}$ of the left-handed chiral helicity and anti-self charge conjugate states $\left|p^{\mu}, \uparrow\right\rangle^{A}$ of the righthanded chiral helicity.

\section{Some Considerations on Interactions}

From a formal point of view, the wave equation (31) may be put in the form $\left(\Gamma^{\mu \nu} p_{\mu} p_{\nu}+m \Gamma^{\mu} p_{\mu}-2 m^{2} \mathbb{1}\right) \lambda\left(p^{\mu}\right)=0$. However, it turns out that $\Gamma^{\mu \nu}$ and $\Gamma^{\mu}$ do not transform as Poincaré tensors. Therefore, the operator that acts on $\lambda\left(p^{\mu}\right)$ in Eq. (31) carries only the indices of the $(1 / 2,0) \oplus(0,1 / 2)$ representation space without the additional structure, which contains contraction[s] of a Poincaré tensor $[\mathrm{s}]$ with an energy momentum four vector $[\mathrm{s}] p_{\mu}$. This has the consequence that gauge interactions cannot be introduced by replacing the $\partial_{\mu}$ by an appropriate gauge-covariant derivative. To understand this result better, let us see what happens to the spinors of type I and type II under simple phase transformation. First, type I spinors: Under the simultaneous transformations $\phi_{R}\left(p^{\mu}\right) \rightarrow e^{i \alpha(x)} \phi_{R}\left(p^{\mu}\right)$ and $\phi_{L}\left(p^{\mu}\right) \rightarrow e^{i \alpha(x)} \phi_{L}\left(p^{\mu}\right)$ on $\phi_{R}\left(p^{\mu}\right)$ and $\phi_{L}\left(p^{\mu}\right)$, the spinors pick up an overall phase factor $e^{i \alpha(x)}$. The demand for covariance of the associated equation of motion (i.e., Dirac equation) under this phase transformation immediately introduces a local $U(1)$ gauge interaction. Next, type II spinors (say the $\lambda\left(p^{\mu}\right)$ spinors): Now let $\phi_{L}\left(p^{\mu}\right) \rightarrow e^{i \alpha(x)} \phi_{L}\left(p^{\mu}\right)$, then $\chi_{R}\left(p^{\mu}\right)$, introduced in (28) and which enters the definition of $\lambda\left(p^{\mu}\right)$, transforms into $e^{-i \alpha(x)} \chi_{R}\left(p^{\mu}\right)$. The transformed $\lambda\left(p^{\mu}\right)$, as is readily seen, is no longer a self/anti-self charge conjugate spinor. These arguments do not rule out the existence of gauge interactions with the particles under consideration, but point to the fact that the gauge interactions (if present) will enter the formalism in a somewhat different fashion.

When the above remarks are considered for spin one half the obvious question arises: What relevance does the self/anti-self charge conjugate $(1 / 2,0) \oplus(0,1 / 2)$ representation space have to neutrinos. How can particles thus described enter gauge interactions? The answer is that within the standard framework the neutrino/anti-neutrino does not belong to the Dirac's $(1 / 2,0) \oplus(0,1 / 2)$ representation space, or the self/anti-self charge conjugate representation space considered here. Neutrino/anti-neutrino are described by the $\left(\mathbb{1} \pm \gamma^{5}\right)$ projectors that project out the left-/right- 
handed Weyl neutrinos [i.e. $(0,1 / 2)$ or $(1 / 2,0)$ representation spaces]. So unless there exist interactions beyond the standard framework without the $\left(\mathbb{1} \pm \gamma^{5}\right)$ projectors involved one cannot distinguish between Diracness (either as a Dirac field or a Majorana field) or the self/anti-self charge conjugateness. If the neutrino participates in interactions beyond the standard model then alone does the construct presented here become relevant. In such a scenario the most natural interaction that arises is of the type $\bar{\nu}(x)\left(\cos \theta+i \gamma^{5} \sin \theta\right) \nu(x) \phi(x)$, where $\phi(x)$ is some scalar field (perhaps of the type suggested by Stephenson and Goldman [32]) and $\theta$ determines the behaviour of the interaction under combined operation of charge conjugation and parity.

\section{Concluding Remarks}

In view of the considerations presented above we conclude that the subject of $(1,3)$ space-time symmetries and its implications for the kinematic structure of quantum field theories is still an open arena. As we have already pointed out, we have not investigated all possible physically relevant requirements that may be used to fix relative phase factors between the $(j, 0)$ and $(0, j)$ spinors. For instance, we may begin with spinors

$$
\psi\left(p^{\mu}\right)=\left(\begin{array}{c}
\phi_{R}\left(p^{\mu}\right) \\
\cos (\theta) \phi_{L}\left(p^{\mu}\right)
\end{array}\right) \text {, or } \quad \psi\left(p^{\mu}\right)=\left(\begin{array}{c}
\cos (\theta) \phi_{R}\left(p^{\mu}\right) \\
\phi_{L}\left(p^{\mu}\right)
\end{array}\right) .
$$

Obviously the kinematic structure derived from these spinors, such as the wave equation, the field operator, and transformation of the physical states under Parity, Charge Conjugation, and Time Reversal, would be very different from those already discussed. Parity is non-maximally violated for $0<\theta<\pi / 2$. Of all the possible kinematic structures, which of these are physically realized in nature depends on what symmetries are respected in Nature and to what extent. Once this question is answered by experimental observations, one may proceed to seek an appropriate kinematic structure to build the interacting theory.

Even though we have not investigated all possible physically relevant requirements that may be used to fix relative phase factors between the $(j, 0)$ and $(0, j)$ spinors our work has been presented in sufficient detail to reduce all such generalizations to conceptually simple and algebraically well defined exercise. It is hoped that such generalizations will be considered in future as necessitated by specific problems at hand.

In summary, we argue that constraints imposed by the kinematic structure on the dynamical aspects of a theory are subtler than the textbook treatment of this subject would indicate. The kinematic symmetries relevant to the problem determine the underlying kinematic structure on which to build one's dynamical theory. Based on the observation that if $\phi_{L}\left(p^{\mu}\right)$ transforms as a $(0, j)$ spinor under Lorentz boosts, then $\Theta_{[j]} \phi_{L}^{*}\left(p^{\mu}\right)$ transforms as a $(j, 0)$ spinor (with a similar relationship existing between $\phi_{R}\left(p^{\mu}\right)$ and $\Theta_{[j]} \phi_{R}^{*}\left(p^{\mu}\right)$ we introduced McLennan-Case type $(j, 0) \oplus$ $(0, j)$ spinors. Relative phases between $\phi_{R}\left(p^{\mu}\right)$ and $\Theta_{[j]} \phi_{R}^{*}\left(p^{\mu}\right)$, and $\Theta_{[j]} \phi_{L}^{*}\left(p^{\mu}\right)$ and $\phi_{L}\left(p^{\mu}\right)$, turn out to have physical significance and are fixed by appropriate requirements. Explicit construction, and a series of physically relevant properties, for these spinors were obtained for spin- $1 / 2$ and spin-1 culminating in the construction of a fundamentally new wave equation and introduction of Dirac-like and Majorana-like quantum fields.

Acknowledgements Apart from the acknowledgements expressed at various places in the text I wish to extend my zimpoic thanks to Christoph Burgard for a series of insightful conversations on 
some of the subject matter of this manuscript. In addition, I thank the following colleagues and friends at the Los Alamos National Laboratory for their constant accessibility and encouragement: George Glass, Terry Goldman, Steve Greene, Peter Herczeg, Cy Hoffman, Mikkel Johnson, Gus Sinnis, and Hywel White; and in particular, all members of the LSND (experimental group studying neutrino oscillations at LAMPF) and MILAGRO (experimental group studying gamma-ray bursts at LANL) for educating and entertaining me with the various mysteries of the Universe during the course of this work. Away from the Laboratory, I enjoyed many conversations on the subject with Rabindra N. Mohapatra at Snowmass '94, and Professor Pierre Ramond kindly directed me to an important paper in the field. I thank them both. The work was indeed inspired by the comment on "magic of the Pauli matrices" (which turned out to be "magic of Wigner's $\Theta_{[j]}$ operator" as we saw in this work) that Professor Pierre Ramond made in his book (Ref. [11, p. 16). Professor Steven Weinberg kindly commented [31] on why Ref. [3] gets "such a surprising result," I wish to thank him for sharing his thoughts on the subject. I wish to thank Jeanne Bowles for a careful reading of the manuscript and for her suggestions on stylistic matters.

This work was done under the auspices of the U. S. Department of Energy. 


\section{TABLES}

TABLE I. For spin-1/2, covariant norms for $\lambda^{S}\left(p^{\mu}\right)$ and $\lambda^{A}\left(p^{\mu}\right)$ for general values of $\vartheta_{1}$ and $\vartheta_{2}$. The value of $\bar{\lambda}_{\eta^{\prime}}\left(p^{\mu}\right) \lambda_{\eta^{\prime \prime}}\left(p^{\mu}\right)$ is tabulated at the intersection of appropriate row and column. a

\begin{tabular}{lcccc}
\hline \hline & $\lambda_{\uparrow}^{S}\left(p^{\mu}\right)$ & $\lambda_{\downarrow}^{S}\left(p^{\mu}\right)$ & $\lambda_{\uparrow}^{A}\left(p^{\mu}\right)$ & $\lambda_{\downarrow}^{A}\left(p^{\mu}\right)$ \\
\hline $\bar{\lambda}_{\uparrow}^{S}\left(p^{\mu}\right)$ & 0 & $2 i N^{2} \cos \left(\vartheta_{1}+\vartheta_{2}\right)$ & 0 & $-2 N^{2} \sin \left(\vartheta_{1}+\vartheta_{2}\right)$ \\
$\bar{\lambda}_{\downarrow}^{S}\left(p^{\mu}\right)$ & $-2 i N^{2} \cos \left(\vartheta_{1}+\vartheta_{2}\right)$ & 0 & $2 N^{2} \sin \left(\vartheta_{1}+\vartheta_{2}\right)$ & 0 \\
$\bar{\lambda}_{\uparrow}^{A}\left(p^{\mu}\right)$ & 0 & $2 N^{2} \sin \left(\vartheta_{1}+\vartheta_{2}\right)$ & 0 & $-2 i N^{2} \cos \left(\vartheta_{1}+\vartheta_{2}\right)$ \\
$\bar{\lambda}_{\downarrow}^{A}\left(p^{\mu}\right)$ & $-2 N^{2} \sin \left(\vartheta_{1}+\vartheta_{2}\right)$ & 0 & $2 i N^{2} \cos \left(\vartheta_{1}+\vartheta_{2}\right)$ & 0 \\
\hline \hline
\end{tabular}

${ }^{\text {a }}$ For the choice of phases $\vartheta_{1}+\vartheta_{2}=0$, we obtain a bi-orthonormal set with sub-spaces spanned by $\lambda^{S}\left(p^{\mu}\right)$ and $\lambda^{A}\left(p^{\mu}\right)$ mutually orthogonal.

TABLE II. For spin-1, covariant norms for $\lambda^{S}\left(p^{\mu}\right)$ and $\lambda^{A}\left(p^{\mu}\right)$ for general values of $\delta_{1}, \delta_{2}$, and $\delta_{3}$. The value of $\bar{\lambda}_{\eta^{\prime}}\left(p^{\mu}\right) \lambda_{\eta^{\prime \prime}}\left(p^{\mu}\right)$ is tabulated at the intersection of appropriate row and column. a

\begin{tabular}{ccccccc}
\hline \hline & $\lambda_{\uparrow}^{S}\left(p^{\mu}\right)$ & $\lambda_{\rightarrow}^{S}\left(p^{\mu}\right)$ & $\lambda_{\downarrow}^{S}\left(p^{\mu}\right)$ & $\lambda_{\uparrow}^{A}\left(p^{\mu}\right)$ & $\lambda_{\rightarrow}^{A}\left(p^{\mu}\right)$ & $\lambda_{\downarrow}^{A}\left(p^{\mu}\right)$ \\
\hline $\bar{\lambda}_{\uparrow}^{S}\left(p^{\mu}\right)$ & 0 & 0 & $\alpha_{+}$ & 0 & 0 & $\alpha_{-}$ \\
$\bar{\lambda}_{\rightarrow}^{S}\left(p^{\mu}\right)$ & 0 & $-\beta_{+}$ & 0 & 0 & $-\beta_{-}$ & 0 \\
$\bar{\lambda}_{\downarrow}^{S}\left(p^{\mu}\right)$ & $\alpha_{+}$ & 0 & 0 & $\alpha_{-}$ & 0 & 0 \\
$\bar{\lambda}_{\uparrow}^{A}\left(p^{\mu}\right)$ & 0 & 0 & $-\alpha_{-}$ & 0 & 0 & $-\alpha_{+}$ \\
$\bar{\lambda}_{\rightarrow}^{A}\left(p^{\mu}\right)$ & 0 & $\beta_{-}$ & 0 & 0 & $\beta_{+}$ & 0 \\
$\bar{\lambda}_{\downarrow}^{A}\left(p^{\mu}\right)$ & $-\alpha_{-}$ & 0 & 0 & $-\alpha_{+}$ & 0 & 0 \\
\hline \hline
\end{tabular}

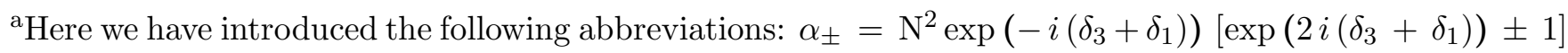
and $\beta_{ \pm}=\mathrm{N}^{2} \exp \left(-2 i \delta_{2}\right)\left[\exp \left(4 i \delta_{2}\right) \pm 1\right]$. Equivalently, $\alpha_{+}=2 \mathrm{~N}^{2} \cos \left(\delta_{3}+\delta_{1}\right), \alpha_{-}=2 i \mathrm{~N}^{2} \sin \left(\delta_{3}+\right.$ $\left.\delta_{1}\right), \beta_{+}=2 \mathrm{~N}^{2} \cos \left(2 \delta_{2}\right)$, and $\beta_{-}=2 i \mathrm{~N}^{2} \sin \left(2 \delta_{2}\right)$. For the choice of phases $\delta_{3}+\delta_{1}=0$ and $\delta_{2}=0$, we obtain a bi-orthonormal set with sub-spaces spanned by $\lambda^{S}\left(p^{\mu}\right)$ and $\lambda^{A}\left(p^{\mu}\right)$ mutually orthogonal. 


\section{REFERENCES}

[1] M. Sawicki and D. V. Ahluwalia, Phys. Lett. B 335, 24 (1994).

[2] L. H. Ryder, Quantum Field Theory (Cambridge University Press, Cambridge, U.K., 1987).

[3] D. V. Ahluwalia, M. B. Johnson, and T. Goldman, Phys. Lett. B 316, 102 (1993).

[4] D. V. Ahluwalia and T. Goldman, Mod. Phys. Lett. A 8, 2623 (1993).

[5] E. P. Wigner, Ann. Math. 40, 149 (1939).

[6] E. Majorana, Nuovo Cimento 14, 171 (1937). For an English translation of this classic work, the reader should refer to technical translation TT-542, National Research Council of Canada. The reader may also contact the author to obtain a copy of the English translation of this and two other Italian language works (Racah's paper [7], and a 1932 paper of Majorana [8]) on the subject for personal use.

[7] G. Racah, Nuovo Cimento 14, 322 (1937). also see C. N. Yang and J. Tiomno, Phys. Rev. 79, 495 (1950).

[8] E. Majorana, Nuovo Cimento 9, 355 (1932). Account in English: D. M. Fradkin, Am. J. Phys. 34, 314 (1966). English translation: C. A. Orzalesi, University of Maryland (Department of Physics and Astronomy) Technical Report number 792 (1968).

[9] J. A. McLennan, Jr., Phys. Rev. 106, 821 (1957).

[10] K. M. Case, Phys. Rev. 107, 307 (1957).

[11] See, for example, P. Ramond, Field Theory: A Modern Primer (Addison-Wesley Publishing Company Inc., California, 1989); and Ref. [2].

[12] H. Jehle, Phys. Rev. 75, 1609 (1949).

[13] A. Athanassopoulos et al., "Candidate Events in Search for $\bar{\nu}_{\mu} \rightarrow \bar{\nu}_{e}$ Oscillations," Los Alamos National Laboratory preprint LA-UR-95-1238.

[14] E. P. Wigner, Z. Phys. 43, 624 (1927).

[15] E. P. Wigner, in Group Theoretical Concepts and Methods in Elementary Particle Physics Physics - Lectures of the Istanbul Summer School of Theoretical Physics, 1962, edited by F. Gürsey;

Also see: Z. K. Silagadze, Yad. Fiz. 55, 707 (1992) [Sov. J. Nucl. Phys. 55, 392 (1992)].

[16] S. Weinberg, Phys. Rev. 133, B1318 (1964).

[17] A. Proca, J. de Phys. et. rad. 7, 347 (1936); R. G. Duffin, Phys. Rev. 54, 1114 (1938); N. Kemmer, Proc. Roy. Soc. (London) A 173, 91 (1939); W. Rartia and J. Schwinger, Phys. Rev. 60, 61 (1941); H. J. Bhabha, Rev. Mod. Phys. 17, 200 (1945); HarishChandra, Phys. Rev. 71, 793 (1947); V. Bargmann and E. P. Wigner, Proc. Nat. Acad. Sci. (USA) 34, 211 (1948); K. Johnson and E. C. G. Sudarshan, Ann. Phys. 13, 126 (1961).

[18] L. L. Foldy, Phys. Rev. 102, 568 (1956).

[19] B. P. Nigam and L. L. Foldy, Phys. Rev. 102, 1410 (1956). Typographical error: In the right hand side of Eq. (37) of this reference $U_{s}(\theta)$ should be corrected to read $U_{s}(\theta=0)$.

[20] O. Nachtmann, Elementary Particle Physics: Concepts and Phenomena (SpringerVerlag, Berlin/Heidelberg, 1990).

[21] For building a $(j, 0) \oplus(0, j)$ Majorana field operator for $j>1 / 2$, one needs counterparts of spin- $1 / 2$ spinors. For explicit expressions of the $(j, 0) \oplus(0, j)$ spinors up to $j=2$, see: D. V. Ahluwalia and D. J. Ernst, Int. J. Mod. Phys. E 2, 397 (1993). The front-form counterparts are cataloged in Ref. [22]. 
[22] D. V. Ahluwalia and M. Sawicki, Phys. Rev. D 47, 5161 (1993).

[23] J. D. Bjorken and S. D. Drell, Relativistic Quantum Mechanics (McGraw-Hill Book Company, New York, 1964).

[24] C. Burgard (private conversations, Summer 1991).

[25] L. H. Ryder (private communication, 1992).

[26] B. Hatfield, Quantum Field Theory of Point Particles and Strings, Frontiers in Physics, Vol. 75 (Addison-Wesley Publishing Company, California, 1992, USA).

[27] E. C. G. Stükelberg, Helv. Phys. Acta 14, 32L (1941); 14, 588 (1941); R. P. Feynman, Phys. Rev. 76, 749 (1949); 76, 769 (1949). For Wheeler's important contribution to this argument, see pp. 387-389 of S. S. Schweber, QED and the Men Who Made it: Dyson, Feynman, Schwinger, and Tomonaga (Princeton University Press, Princeton, 1994, USA).

[28] P. A. M. Dirac, Proc. Roy. Soc. Lond. A 322, 435 (1971); 328, 1 (1972).

[29] P. A. M. Dirac, Directions in Physics (John Wiley \& Sons, New York, 1978, USA).

[30] H. Joos, Fortschr. Physik 10, 65 (1962).

[31] S. Weinberg (private communication, 1993).

[32] G. S. Stephenson, Jr. and T. Goldman, "Observable Consequences of a Scalar Boson Coupled Only to Neutrions, "Los Alamos National Laboratory Preprint LA-UR-93-3348; hep-ph/9309308. 